\title{
Comparison of sequential therapy and amoxicillin/tetracycline containing bismuth quadruple therapy for the first-line eradication of Helicobacter pylori: a prospective, multi-center, randomized clinical trial
}

Ju Yup Lee ${ }^{1,2}$, Nayoung Kim ${ }^{1 *}$, Kyung Sik Park², Hyun Jin Kim³ , Seon Mee Park , Gwang Ho Baik ${ }^{5}$, Ki-Nam Shim , Jung Hwan Oh${ }^{7}$, Suck Chei Choi ${ }^{8}$, Sung Eun Kim', Won Hee Kim ${ }^{10}$, Seon-Young Park ${ }^{11}$, Gwang Ha Kim², Bong Eun Lee ${ }^{12}$, Yunju Jo $\mathrm{O}^{13}$ and Su Jin Hong ${ }^{14}$

\begin{abstract}
Background: The $<80 \%$ Helicobacter pylori eradication rate with sequential therapy is unsatisfactory. Modified bismuth quadruple therapy, replacing metronidazole with amoxicillin, could be promising because $\mathrm{H}$. pylori resistance to tetracycline or to amoxicillin is relatively low. A 14-day modified bismuth quadruple protocol as firstline H. pylori treatment was compared with 10-day sequential therapy.

Methods: In total, $390 \mathrm{H}$. pylori-infected subjects participated in the randomized clinical trial: 10-day sequential therapy (40 mg pantoprazole plus $1 \mathrm{~g}$ amoxicillin twice a day for 5 days, then $40 \mathrm{mg}$ pantoprazole and $500 \mathrm{mg}$ clarithromycin twice a day and 500 mg metronidazole three times a day for 5 days) or 14-day modified bismuth quadruple therapy (40 mg pantoprazole, $600 \mathrm{mg}$ bismuth subcitrate, $1 \mathrm{~g}$ tetracycline, and $1 \mathrm{~g}$ amoxicillin, twice a day). ${ }^{13} \mathrm{C}$-urea breath test, rapid urease testing, or histology was performed to check for eradication.

Results: Intention-to-treat (ITT) eradication rates of 10-day sequential and 14-day quadruple therapy were $74.6 \%$ and $68.7 \%$, respectively, and the per-protocol (PP) rates were 84.2 and $76.5 \%$, respectively. The eradication rate was higher in the sequential therapy group, but neither the ITT nor the PP analyses had a significant difference $(P=0.240$ and $P=0.099$, respectively). However, the adverse events were significantly lower in the modified bismuth quadruple therapy group than the sequential therapy group (36.9 vs. $47.7 \%, P=0.040$ ).

Conclusions: Ten-day sequential therapy appears to be more effective despite frequent adverse events. However, both 10-day SQT and 14-day PBAT did not reach the excellent eradication rates that exceed $90 \%$. Additional trials are needed to identify a more satisfactory first-line eradication therapy.
\end{abstract}

Trial registration: ClinicalTrials.gov (NCT02159976); Registration date: 2014-06-03, CRIS (KCT0001176); Registration date: 2014-07-23.

Keywords: Helicobacter pylori, Eradication, Amoxicillin, Tetracycline, Bismuth, Quadruple

* Correspondence: nayoungkim49@empas.com

'Department of Internal Medicine, Seoul National University Bundang

Hospital, Seongnam, South Korea

Full list of author information is available at the end of the article 


\section{Background}

Helicobacter pylori is a major cause of gastric diseases such as chronic gastritis, gastroduodenal ulcers, and gastric cancer, and it is well known that the eradication of $H$. pylori is important in preventing and treating these gastric diseases $[1,2]$. Recently, the Kyoto Global Consensus Meeting, held in Japan in early February of 2014, presented radical changes in the diagnosis and treatment of $H$. pylori infection, such as recommending eradication treatment for patients with dyspepsia [3]. In addition, eradication therapy was recommended for all $H$. pylori-positive individuals for the purpose of preventing $H$. pylori-related diseases [3]. However, the eradication rates of first-line triple therapy, which consists of a proton pump inhibitor (PPI) and two antibiotics (clarithromycin and amoxicillin), have been continuously decreasing [4]. Only $\sim 18 \%$ of previous studies have reported exceeding $85 \%$ eradication on an intention-to-treat (ITT) analysis, with $\sim 60 \%$ falling short of $80 \%$ [5]. The reason for the decrease in the efficacy of PPI-based triple therapy is mainly due to the increase in $H$. pylori resistance to clarithromycin. As such, in Western countries, standard triple therapy is currently considered a "legacy therapy" [5]. In fact, recent European guidelines recommended triple therapy as the first-line treatment only when the prevalence of clarithromycin resistance is under $20 \%$ [6].

To overcome this unsatisfactory eradication rate, sequential therapy (SQT) is currently recommended as an alternative first-line treatment for $H$. pylori infection. Many European randomized clinical trials (RCTs) and meta-analyses have shown the superiority of SQT over standard PPI-based triple therapy [7-9]. In a Korean meta-analysis based on six RCTs, the eradication rate of SQT was $79.4 \%$ in ITT analysis and $86.4 \%$ in PP analysis; this meta-analysis also proved that SQT is superior to standard PPI-based triple therapy (relative risk [RR] 1.761, $95 \%$ confidence interval [CI]; 1.403-2.209) [10]. However, the eradication rate of SQT in Korea, mostly under 80 \% (ITT: 79.4 \%, 95 \% CI: 76.3-82.2) [10], is not satisfactory and is about $10 \%$ lower than reported in early European studies [10,11]. This suboptimal eradication rate could be explained by high rates of antibiotic resistance, especially to clarithromycin, metronidazole, or both [12]. Previous studies showed that the rate of resistance to clarithromycin was $>20 \%$, that to metronidazole was $>30 \%$, and that to both was $>10 \%[13,14]$.

The classical bismuth quadruple therapy, which consists of a PPI, bismuth, tetracycline, and metronidazole, is frequently used as a first- or second-line regimen [6, 15-17]. However, it is known that more than $30 \%$ of patients stop taking their medicine due to the complicated regimen and the high rate of adverse events [18]. In view of antibiotic resistance, a modified bismuth quadruple therapy (PBAT), in which metronidazole is replaced with amoxicillin, is very attractive because many studies have reported that $H$. pylori resistance to amoxicillin and tetracycline is low [19] and furthermore, amoxicillin is easier to take in comparison to metronidazole. Several studies have suggested good eradication rates with this amoxicillin and tetracycline combined quadruple regimen [20-23]; however, the reported efficacies are conflicting. From this background the aim of this study was to evaluate the efficacy of 14-day PBAT as a first-line treatment and compare 10-day SQT to 14-day PBAT in order to establish a more effective first-line regimen for $H$. pylori in terms of eradication rate and adverse effects.

\section{Methods}

\section{Study design and participants}

A prospective, multi-center, randomized, open-label, parallel design clinical trial was conducted between July 2014 and May 2015 in 14 tertiary hospitals from different regions in Korea. Inclusion criteria were adult Korean men and women (aged $\geq 18$ years) who were diagnosed as $H$. pyloripositive by any of the following three methods: 1) a positive rapid urease test (CLOtest [Delta West, Bentley, Australia]), 2) histologic evidence of $H$. pylori by modified Giemsa staining, or a 3) positive ${ }^{13} \mathrm{C}$-urea breath test. Exclusion criteria were 1) age under 18 years; 2) previous eradication therapy for $H$. pylori; 3) drugs that could influence the study results such as a PPI, $\mathrm{H}_{2}$ blocker, mucosal protective agent, or antibiotics within the prior 4 weeks; 3) previous gastric surgery; 4) advanced gastric cancer or other malignancy; 5) abnormal liver function or liver cirrhosis; 6) abnormal renal function or chronic kidney disease; 7) other severe concurrent diseases; 8) previous contraindications or allergic reactions to the study drugs; 9) genetic disorders such as galactose intolerance, the Lapp lactase deficiency, or glucose-galactose malabsorption; 10) mental disorders or alcohol or drug addiction; 11) pregnancy or lactation or refusal to use an appropriate method of contraception throughout the course of the study; 12) any condition that might affect the evaluation of the clinical results in the judgment of the principal investigator or sub-investigator; or 13) any specific contraindication to the study drugs.

The study protocol was approved by the Korean Food and Drug Administration (KFDA No. 30157) and by the Institutional Review Board and Ethics Committees of all participating hospitals (Additional file 1). The study was performed according to Good Clinical Practices (GCP) and the Declaration of Helsinki, and written informed consent was obtained from all patients before enrollment. In addition, this study protocol has been registered at ClinicalTrials.gov (NCT02159976) and Clinical Research Information Service (CRIS) (KCT0001176).

\section{Randomization and treatment allocation}

An independent statistician at Seoul National University Bundang Hospital (SNUBH) prepared the randomization. 
The subjects were randomized in a 1:1 ratio by a block randomization method to receive either 10-day SQT or 14-day PBAT. The 10-day SQT consisted of $40 \mathrm{mg}$ of pantoprazole plus $1 \mathrm{~g}$ of amoxicillin twice a day for the initial 5 days, followed by $40 \mathrm{mg}$ of pantoprazole and $500 \mathrm{mg}$ of clarithromycin twice a day and $500 \mathrm{mg}$ of metronidazole three times a day for the subsequent 5 days. The 14-day PBAT consisted of $40 \mathrm{mg}$ of pantoprazole, $600 \mathrm{mg}$ of bismuth subcitrate, $1 \mathrm{~g}$ of tetracycline, and $1 \mathrm{~g}$ of amoxicillin, twice a day. Four weeks after completing the therapy, successful $H$. pylori eradication was defined by a negative ${ }^{13} \mathrm{C}$ urea breath test or an invasive test when endoscopic follow-up was needed in cases of benign gastric ulcer. The drug compliance and adverse events were evaluated by a physician via direct questioning. Compliance was considered to be satisfactory when drug intake exceeded $85 \%$.

\section{Assessment of $H$. pylori infection}

\section{Invasive $H$. pylori test (Giemsa histology and CLOtest)}

To determine the presence of current $H$. pylori infection, four biopsy specimens (one each from the greater curvature and lesser curvature of the antrum and body) were taken from the gastric mucosa at each endoscopic examination and were fixed in formalin to be used for the evaluation of $H$. pylori infection by Giemsa staining [24]. Two specimens from the lesser curvature of the antrum and body were used for the rapid urease test (CLOtest) [24].

\section{${ }^{13} \mathrm{C}$-urea breath test}

Subjects were fasted for $4 \mathrm{~h}$ prior to testing and a predose breath sample was obtained; $100 \mathrm{mg}$ of ${ }^{13} \mathrm{C}$-urea powder (UBiTkit; Otsuka Pharmaceutical Co. Ltd., Tokyo, Japan) dissolved in $100 \mathrm{~mL}$ of water was then administered orally, and a second breath sample was collected 20 min later. The cutoff value was $2.5 \%$. The collected samples were analyzed using an isotope ratio mass spectrometer (UBiT-IR300; Otsuka Pharmaceutical Co., Ltd.).

\section{Trial outcomes}

The primary outcome was comparing the percentage of participants with successful $H$. pylori eradication in the 10-day SQT and 14-day PBAT groups 4-6 weeks after completion of eradication therapy. The secondary outcome was to compare the percentage of patients whose drug compliance was greater than $85 \%$ and the percentage of adverse events in the 10-day SQT and 14-day PBAT groups.

\section{Sample size and statistical analysis}

Because the eradication rate of the 10-day SQT was found to be $82.0 \%$ in a previous Korean report [10] the eradication rate of the 14-day PBAT was assumed to be similar to that of the 10-day SQT if the rate difference between the two regimens was less than $10 \%$. With a power of $80 \%$ at a two-sided type 1 error rate of $5 \%, 195$ subjects were needed for each treatment arm to allow for $10 \%$ loss to follow-up. The H. pylori eradication rate was determined by both an ITT and a per-protocol (PP) analysis. All subjects who received treatment were included in the ITT analysis. For the PP analysis, subjects who were lost to follow-up, had taken less than $85 \%$ of the prescribed drugs, or had dropped out due to severe adverse events were excluded.

Parametric continuous variables were compared using the Student's $t$-test and are presented as mean \pm standard deviation (SD). Categorical variables were analyzed using Pearson's chi square test or Fisher's exact test and were presented as numbers (percentages). Univariate and multivariate logistic regression were used for analysis of influencing factors, which were expressed as the odds ratios (OR) and $95 \%$ confidence intervals (CI). A two-sided $P$ value of less than 0.05 was considered statistically significant. All statistical analyses were performed using SPSS (version 20.0; SPSS Inc., Chicago, IL, US).

\section{Results \\ Subjects}

A total of $390 \mathrm{H}$. pylori-infected treatment-naïve subjects were randomly assigned to the 10 -day SQT group $(n=195)$ or the 14-day PBAT group $(n=195)$ (Fig. 1). Nineteen subjects in the SQT group and 18 subjects in the PBAT group did not complete the study due to adverse events, loss of follow-up, or withdrawal of consent. Therefore, 176 subjects in the SQT group and 175 subjects in the PBAT group completed the follow-up. After exclusion of 11 and 7 noncompliant subjects who consumed $<85 \%$ of the prescribed medications, 165 and 170 subjects in the SQT and PBAT groups, respectively, became the objects of the PP analysis (Fig. 1). There were no significant differences in the baseline characteristics or endoscopic findings between the two groups (Table 1).

\section{Eradication rates}

ITT eradication rates of the 10-day SQT and 14-day PBAT groups were $74.6 \%(146 / 195)$ and $68.7 \%(134 / 195)$ and the PP eradication rates were $84.2 \%(139 / 165)$ and $76.5 \%$ (130/170), respectively. The eradication rate was higher in the SQT than the PBAT group, but there was no statistical significance on either ITT or PP analysis $(P=0.240$ and $P=0.099$, respectively) (Fig. 2). There also was no statistical significance in the ITT analysis conducted independently at each institution (Fig. 3).

\section{Compliance and adverse events}

The complete follow-up rate was $90.3 \%(176 / 195)$ in the 10-day SQT and $90.8 \%$ in the 14-day PBAT group. The numbers of subjects who took more than $85 \%$ of the prescribed medicine were $93.8 \%(165 / 176)$ in the 


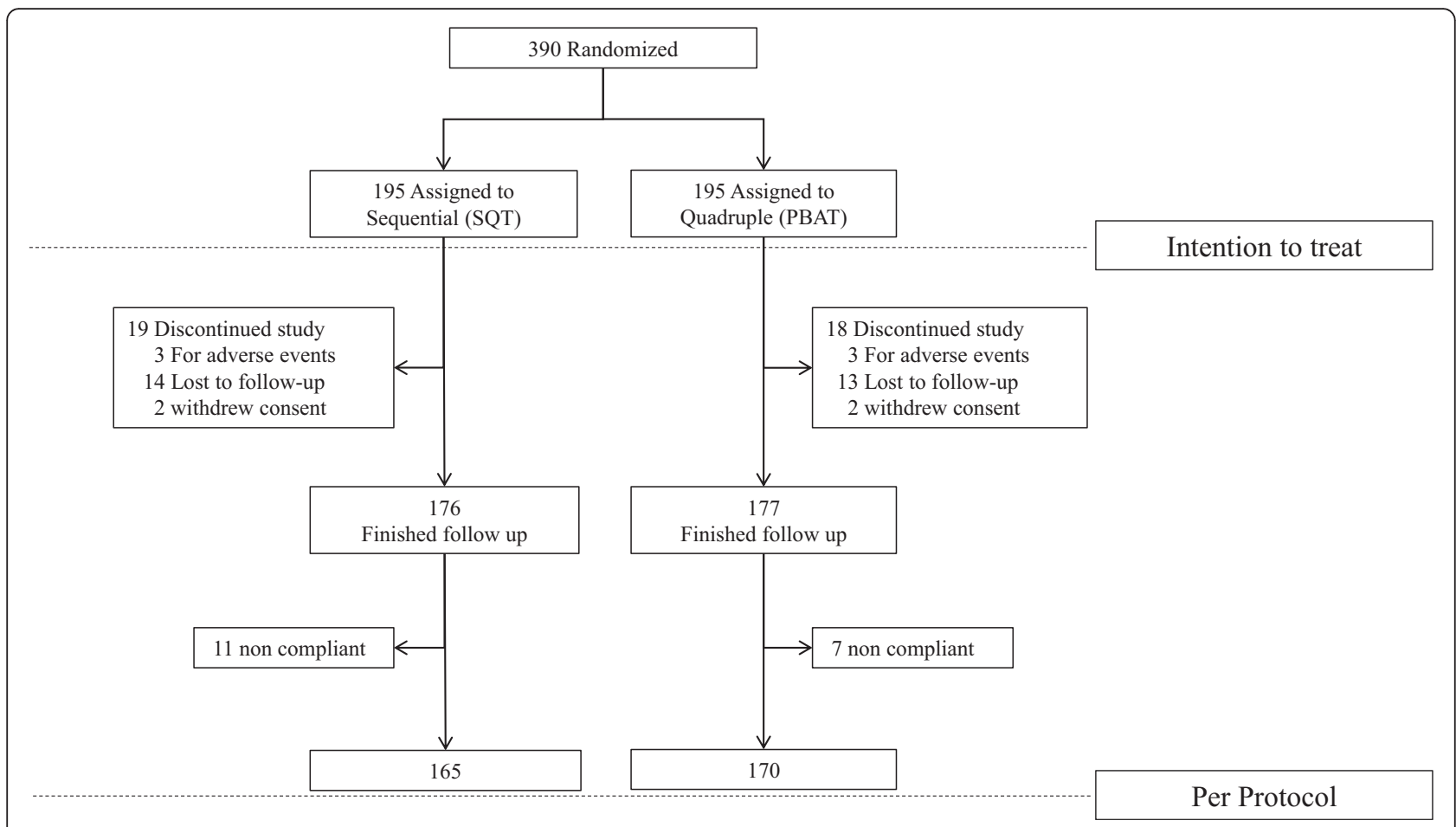

Fig. 1 Flowchart of participants

Table 1 Baseline characteristics of the subjects

\begin{tabular}{llll}
\hline & SQT $(n=195)$ & PBAT $(n=195)$ & $p$-value \\
\hline Gender, n (\%) & & & 0.184 \\
Male & $103(52.8)$ & $117(60.0)$ & \\
$\quad$ Female & $92(47.2)$ & $78(40.0)$ & \\
Age (mean \pm SD), year & $53.1 \pm 12.6$ & $53.6 \pm 13.2$ & 0.697 \\
BMl, kg/m ${ }^{2}$ & $23.5 \pm 3.1$ & $23.9 \pm 3.4$ & 0.328 \\
Smoking, n (\%) & $38(19.5)$ & $36(18.5)$ & 0.897 \\
Alcohol, n (\%) & $75(38.5)$ & $72(36.9)$ & 0.835 \\
Endoscopic finding, n (\%) & & & \\
$\quad$ Normal & $10(5.1)$ & $11(5.6)$ & 1.000 \\
Atrophic gastritis with or & $43(22.1)$ & $31(15.9)$ & 0.155 \\
without intestinal metaplasia & & & \\
Other gastritis & $12(6.2)$ & $16(8.2)$ & 0.556 \\
Gastric ulcer & $47(24.1)$ & $43(22.1)$ & 0.718 \\
Duodenal ulcer & $15(7.7)$ & $20(10.3)$ & 0.479 \\
Gastric ulcer + Duodenal & $4(2.1)$ & $7(3.6)$ & 0.541 \\
ulcer & & & \\
EMR, ESD for dysplasia & $16(8.2)$ & $18(9.2)$ & 0.858 \\
Or EGC & $1(0.5)$ & $0(0.0)$ & 1.000 \\
MALToma & $1(0.5)$ & $3(1.5)$ & 0.615 \\
Reflux esophagitis & $10(5.1)$ & $7(3.6)$ & 0.620 \\
Others & & & \\
\hline
\end{tabular}

SQT sequential therapy, PBAT quadruple therapy consist of pantoprazole, bismuth, amoxicillin, and tetracycline, $B M I$ body mass index, EMR endoscopic mucosal resection, $E S D$ endoscopic submucosal dissection, $E G C$ early gastric cancer, MALToma mucosal associated lymphoid tissue lymphoma 10-day SQT group and $96.1 \%(170 / 177)$ in the 14-day PBAT group. There was also no significant difference between the two groups $(P=0.460)$. Ninety-three patients $(47.7 \%)$ who took the sequential regimen and 72 patients $(36.9 \%)$ who took the quadruple regimen experienced at least one adverse event. The adverse event rate was significantly lower in the PBAT group than in the SQT group $(P=0.040)$. In the 10 -day SQT group, the most frequent adverse events were taste distortion (25.8\%) and abdominal bloating (22.6\%). In the 14-day PBAT group, the most frequent adverse events were epigastric discomfort (23.6 \%) and diarrhea (16.7\%). Taste distortion was more frequently reported in the 10-day SQT group $(P<0.001)$ and diarrhea was more frequently reported in the 14-day PBAT group $(P=0.014)$ (Table 2$)$.

\section{Factors associated with eradication failure in PBAT}

On univariate analysis, male sex (OR, 0.38; $95 \% \mathrm{CI}$, $0.31-1.30)$ and $\mathrm{BMI} \geq 25 \mathrm{~kg} / \mathrm{m}^{2}$ (OR, 2.28; $95 \% \mathrm{CI}$, 1.12-4.63) were associated with treatment failure in the 14-day PBAT group. The multivariate analysis confirmed that both male sex (OR, 0.27; $95 \% \mathrm{CI}, 0.34-1.51)$ and BMI (OR, 2.16; 95 \% CI, 1.05-4.43) were associated with treatment failure in the 14-day PBAT group (Table 3).

\section{Discussion}

Antibiotic resistance is one of the main causes of treatment failure in $H$. pylori eradication. The rates of clarithromycin 


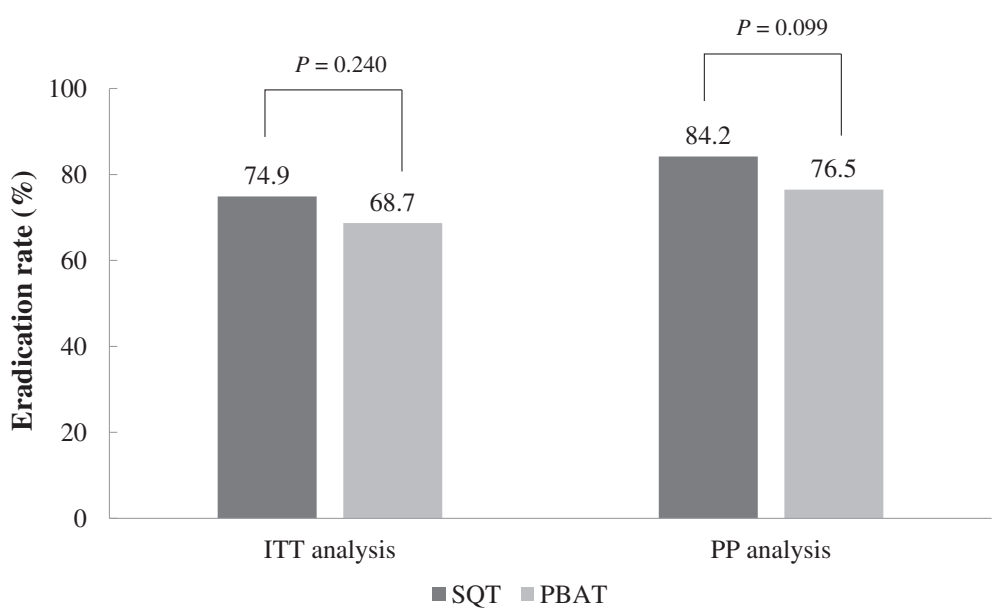

Fig. $2 \mathrm{H}$. pylori eradication rate of 10-day SQT and 14-day PBAT according to the ITT and PP analyses. SQT, sequential therapy; PBAT, quadruple therapy consists of pantoprazole, bismuth, amoxicillin, and tetracycline; ITT, intention-to-treat; PP, per-protocol

resistance increased rapidly from 23.2 to $37.3 \%$ from 2003 to 2012 and the metronidazole resistance rate was $35.8 \%$ between 2009 and 2012 in Korea [4]. In contrast, the rates of resistance to amoxicillin and tetracycline are relatively low, at 17.2 and $10.8 \%$, respectively [4]. The average resistance rate to amoxicillin in Europe is reported to be lower than $2 \%$, and the resistance rate to tetracycline is reported to be below $5 \%$ in most countries [25-27].
We hypothesized that a quadruple regimen containing amoxicillin and tetracycline would demonstrate a superior eradication rate and could be a suitable substitute for triple or sequential regimens in the first-line treatment of H. pylori infection. The ITT eradication rate of the 14-day PBAT was $68.7 \%$ and that of 10-day SQT was $74.6 \%$. There was no statistically significance difference between these two treatment groups $(P=0.240)$, suggesting that

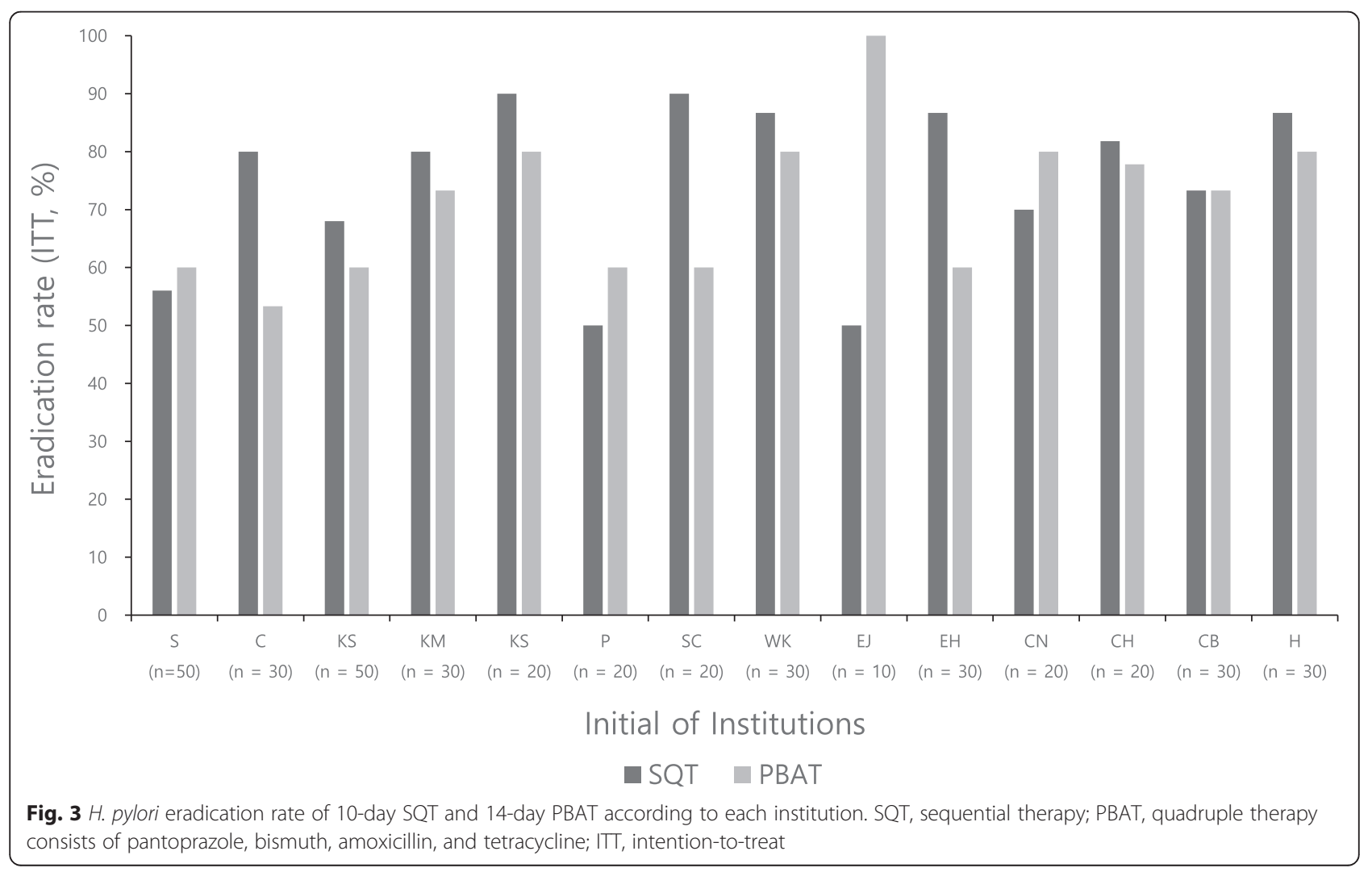


Table 2 Adverse events of the subjects

\begin{tabular}{llll}
\hline & SQT $(n=195)$ & PBAT $(n=195)$ & $p$-value \\
& $\mathrm{n}(\%)$ & $\mathrm{n}(\%)$ & \\
\hline Bloating & $21(22.6)$ & $11(15.3)$ & 0.097 \\
Epigastric soreness & $15(16.1)$ & $17(23.6)$ & 0.854 \\
Anorexia & $2(2.2)$ & $1(1.4)$ & 1.000 \\
Taste distortions & $24(25.8)$ & $4(5.6)$ & $<0.001$ \\
Nausea & $14(15.1)$ & $6(8.3)$ & 0.108 \\
Vomiting & 0 & $2(2.8)$ & 0.478 \\
Abdominal pain & $4(4.3)$ & $4(5.6)$ & 0.721 \\
Headache & $1(1.1)$ & $2(2.8)$ & 1.000 \\
Dyspepsia & $4(4.3)$ & $4(5.6)$ & 0.721 \\
Diarrhea & $2(2.2)$ & $12(16.7)$ & $\mathbf{0 . 0 1 4}$ \\
Constipation & $2(2.2)$ & $3(4.2)$ & 1.000 \\
Reflux & $1(1.1)$ & $1(1.4)$ & 0.478 \\
Rash and itching & $2(2.2)$ & $5(2.8)$ & 0.446 \\
Dizziness & $1(1.1)$ & 0 & 1.000 \\
Stool color change & 0 & $3(4.2)$ & 0.246 \\
Total & $93(47.7)$ & $72(36.9)$ & $\mathbf{0 . 0 4 0}$ \\
\hline
\end{tabular}

Bold style, means statistical significance

SQT sequential therapy, PBAT quadruple therapy consist of pantoprazole, bismuth, amoxicillin, and tetracycline

14-day PBAT was not inferior to 10-day SQT and therefore 14-day PBAT could be another treatment option.

However, some controversies in the results and large heterogeneity in the duration or the first- or second-line regimen exists in previous studies of amoxicillin- and tetracycline-containing quadruple regimens. For instance, in a study of the 14-day quadruple regimen, the eradication rate was $33.3 \%$ in amoxicillin-susceptible $H$. pylori-infected patients [28]. In contrast, a Chinese study showed that the eradication rate of 14-day LBAT (lansoprazole, bismuth, amoxicillin, and tetracycline) was 83.8 \% (95 \% CI: 76.8-90.9 \%) [20]. Furthermore, an RCT performed in Turkey reported the eradication rate of 14-day EBAT (esomeprazole, bismuth, amoxicillin, and tetracycline) as $79.0 \%$ (95 \% CI: 71-87 \%) [21]. Chi et al. [23] reported that quadruple therapy containing amoxicillin and tetracycline is an effective regimen to rescue patients after a failed triple therapy by overcoming the antimicrobial resistance of $H$. pylori with an eradication rate of $78 \%$ in ITT and $89 \%$ in PP.

Since $H$. pylori resistance to amoxicillin and tetracycline is uncommon, the explanation for the heterogeneous results could be that both amoxicillin and tetracycline are weak against $H$. pylori or possibly there is an antagonistic effect. However, any possible antagonistic effect between these two antibiotics is unclear and it is difficult to elucidate because data on the gastric bioavailability of tetracycline is lacking [29]. Furthermore, several studies showed a good eradication rate (78.0-83.8 \%), which was not inferior to other regimens, and this positive tendency was also proven in a recent meta-analysis. Published in 2015, the meta-analysis, which included 9 RCTs, showed that the total eradication rate of a quadruple regimen containing amoxicillin and tetracycline was $78.1 \%$ in ITT and $84.5 \%$ in PP, which was not inferior to other quadruple regimens (pooled odds ratio [OR]: 0.9, 95 \% CI: 0.42-1.78) [30]. In subgroup analysis, the eradication rates of 7-day, 10-day, and 14-day amoxicillin/tetracycline quadruple regimens were $67.4,84.6$, and $82.3 \%$, respectively, and the pooled OR of an amoxicillin/tetracycline quadruple regimen used as first-line therapy was 2.34 (95 \% CI: 0.74-7.42) [30].

In our study, we used different doses and intervals of PBAT compared with those of previous studies to maximize subject compliance. In previous studies, bismuth was administered at $300 \mathrm{mg}$ and tetracycline at $500 \mathrm{mg}$, both four times a day [21,31]. The mixture of twice a day and four times a day can influence drug compliance. A recently developed three-in-one capsule containing bismuth, tetracycline, and metronidazole improved the eradication rate of bismuth quadruple therapy over $90 \%$ by reducing the number of medicines and therefore improving patient compliance [32]. In the present clinical trial bismuth $600 \mathrm{mg}$ twice a day and tetracycline $1000 \mathrm{mg}$ twice a day were chosen to increase compliance.

Tetracycline is a time-dependent (half-life 8-10 h) antibiotic agent and has a long post-antibiotic effect (PAE) [33]. Even though a high blood concentration of

Table 3 Factors associated with eradication failure in amoxicillin- and tetracycline containing quadruple therapy

\begin{tabular}{|c|c|c|c|c|}
\hline Factors & $\begin{array}{l}\text { Crude } \\
\text { OR }(95 \% \text { Cl) }\end{array}$ & $\begin{array}{l}\text { Univariated } \\
p \text {-value }\end{array}$ & $\begin{array}{l}\text { Adjusted } \\
\text { OR }(95 \% \mathrm{Cl})^{\mathrm{b}}\end{array}$ & $\begin{array}{l}\text { Multivariated } \\
p \text {-value }\end{array}$ \\
\hline Age $\geq 50$ (y) & $1.12(0.54-2.32)$ & 0.763 & & \\
\hline Female & $0.38(0.31-1.30)$ & $<0.0001$ & $0.27(0.34-1.51)$ & $<0.0001$ \\
\hline Cigarette smoking & $1.35(0.57-3.20)$ & 0.499 & & \\
\hline Alcohol drinking & $1.42(0.70-2.88)$ & 0.325 & & \\
\hline $\mathrm{BMI} \geq 25\left(\mathrm{~kg} / \mathrm{m}^{2}\right)$ & $2.28(1.12-4.63)$ & 0.023 & $2.16(1.05-4.43)$ & 0.037 \\
\hline
\end{tabular}

$O R$ odds ratio, $\mathrm{Cl}$ confidence intervals, $\mathrm{BMI}$ body mass index

annivariate logistic regression

${ }^{\mathrm{b}}$ Adjusted for gender and BMI

${ }^{\mathrm{C}}$ Multivariate logistic regression 
tetracycline does not increase its sterilizing power, it can inhibit bacterial regrowth for a longer period of time $[34,35]$. From these pharmacokinetic considerations, a twice-a-day regimen is also appropriate and is likely to promote patient compliance. The drug compliance in the present study was $96.1 \%$ in the PBAT group, which is comparable to that in the SQT group (93.8\%). In addition, this rate is higher than the $88.6 \%$ [20] and $92.0 \%$ [31] reported by other studies.

This clinical trial had some limitations. First, H. pylori culture was not routinely performed and we could not evaluate the eradication rate of amoxicillin- and/or tetracycline-susceptible $H$. pylori strains. However, one center (SNUBH) performed H. pylori cultures and antibiotic susceptibility testing by the agar dilution method in a small number of patients. The first-line or second-line eradication rate of PBAT was $57.1 \%(8 / 14)$ in amoxicillinand tetracycline-susceptible subjects (data not shown). More antibiotic susceptibility data are needed in a future study to confirm the eradication effect of amoxicillin and tetracycline-containing regimens both in vitro and in vivo. Second, the limited accuracy of ${ }^{13} \mathrm{C}$-UBT at 4-6 weeks after the completion of a bismuth-based treatment that makes possible, at least in part, a false negative diagnosis of $H$. pylori eradication leading to an overestimation of success rates with the bismuth-based regimen (PBAT). However, ${ }^{13} \mathrm{C}$-UBT is a convenient non-invasive method for $H$. pylori diagnosis and popularly used for eradication success. It is very difficult to perform invasive $H$. pylori test such as histology or CLOtest via upper endoscopy to all patients in real clinical setting who did not need follow-up endoscopy and medical cost is also a problem. Third, we cannot elucidate the reason why PBAT showed a lower eradication rate than expected. However, there is a fairly large eradication rate gap between positive and negative studies. Further study is needed in the future.

\section{Conclusions}

In conclusion, this prospective, multi-center, randomized, open-label, parallel design clinical trial demonstrated that SQT for 10 days appears more effective than PBAT in spite of frequent adverse events. However, both 10-day SQT and 14-day PBAT did not achieve excellent eradication rates $(>90 \%)$. Thus, additional trials are necessary to identify a more satisfactory first-line eradication therapy for H. pylori.

\section{Additional file}

Additional file 1: Lists of the IRBs. (DOCX $15 \mathrm{~kb}$ )

\section{Abbreviations}

EGC, early gastric cancer; EMR, endoscopic mucosal resection; ESD, endoscopic submucosal dissection; H. pylori, Helicobacter pylori; MALToma, mucosal associated lymphoid tissue lymphoma; PBAT, quadruple therapy consist of pantoprazole, bismuth, amoxicillin, and tetracycline; SQT, sequential therapy

\section{Acknowledgements}

This work was supported by grant no 06-2014-074 from the Seoul National University Bundang Hospital Research. The authors thank Division of Statistics in Medical Research Collaborating Center at Seoul National University Bundang Hospital for providing statistical consultation.

\section{Availability of data and materials}

Data will not be made available in order to protect the participant identity.

\section{Authors' contributions}

JYL reviewed the literature and drafted the manuscript; NK designed and supervised research; KSP reviewed draft and performed research; HJK and GHK advised and performed research; SMP, GHB, KNS, JHO, SCC, SEK, WHK, SYP, BEL, JYJ, SJH performed clinical procedures including endoscopies, patient enroll, and data collection. JYL and SEK analyzed data. All authors read and approved the final manuscript.

\section{Competing interests}

The authors declare that they have no competing interests.

\section{Consent for publication}

Not applicable.

\section{Ethics approval and consent to participate}

This study was performed according to the Declaration of Helsinki. Written consent was required from all patients. The study protocol was approved by the Korean Food and Drug Administration (KFDA No. 30157) and by the Institutional Review Board and Ethics Committees of all participating hospitals (Additional file 1).

\section{Author details}

${ }^{1}$ Department of Internal Medicine, Seoul National University Bundang Hospital, Seongnam, South Korea. ${ }^{2}$ Department of Internal Medicine, Keimyung University School of Medicine, Daegu, South Korea. ${ }^{3}$ Department of Internal Medicine and Institute of Health Science, Gyeongsang National University School of Medicine, Jinju, Gyeongsangnam-do, South Korea. ${ }^{4}$ Department of Internal Medicine, College of Medicine, Chungbuk National University, Cheongju, South Korea. ${ }^{5}$ Department of Internal Medicine, Hallym University College of Medicine, Chuncheon Sacred Heart Hospital,

Chuncheon, South Korea. ${ }^{6}$ Department of Internal Medicine, Ewha Womans University School of Medicine, Seoul, South Korea. ${ }^{7}$ Departments of Internal Medicine, College of Medicine, The Catholic University of Korea, Seoul, Republic of Korea. ${ }^{8}$ Department of Internal Medicine, Wonkwang University School of Medicine, Iksan, South Korea. ${ }^{9}$ Department of Internal Medicine, Kosin University College of Medicine, Busan, South Korea. ${ }^{10}$ Digestive Disease Center, CHA Bundang Medical Center, CHA University, Seongnam, South Korea. ${ }^{11}$ Department of Internal Medicine, Chonnam National University Medical School, Gwangju, South Korea. ${ }^{12}$ Department of Internal Medicine, Pusan National University School of Medicine and Biomedical Research Institute, Pusan National University Hospital, Busan, South Korea.

${ }^{13}$ Department of Internal Medicine, Eulji General Hospital, Eulji University School of Medicine, Seoul, South Korea. ${ }^{14}$ Department of Internal Medicine and Research Institute, Soonchunhyang University College of Medicine, Bucheon, South Korea.

Received: 22 December 2015 Accepted: 14 July 2016

Published online: 26 July 2016

References

1. McColl KE. Clinical practice. Helicobacter pylori infection. New Engl J Med. 2010:362:1597-604.

2. Graham DY, Lu H, Yamaoka Y. A report card to grade Helicobacter pylori therapy. Helicobacter. 2007;12:275-8.

3. Sugano K, Tack J, Kuipers EJ, Graham DY, El-Omar EM, Miura S, Haruma K, Asaka M, Uemura N, Malfertheiner $\mathrm{P}$, et al. Kyoto global consensus report on Helicobacter pylori gastritis. Gut. 2015;64:1353-67.

4. Lee JY, Kim N, Kim MS, Choi YJ, Lee JW, Yoon H, Shin CM, Park YS, Lee DH, Jung HC. Factors affecting first-line triple therapy of Helicobacter pylori 
including CYP2C19 genotype and antibiotic resistance. Dig Dis Sci. 2014;59:1235-43.

5. Graham DY, Fischbach L. Helicobacter pylori treatment in the era of increasing antibiotic resistance. Gut. 2010;59:1143-53.

6. Malfertheiner P, Megraud F, O'Morain CA, Atherton J, Axon AT, Bazzoli F, Gensini GF, Gisbert JP, Graham DY, Rokkas T, et al. Management of Helicobacter pylori infection-the Maastricht IV/Florence Consensus Report. Gut. 2012;61:646-64.

7. Jafri NS, Hornung CA, Howden CW. Meta-analysis: sequential therapy appears superior to standard therapy for Helicobacter pylori infection in patients naive to treatment. Annals Intern Med. 2008;148:923-31.

8. Gatta L, Vakil N, Leandro G, Di Mario F, Vaira D. Sequential therapy or triple therapy for Helicobacter pylori infection: systematic review and meta-analysis of randomized controlled trials in adults and children. Am J Gastroenterol. 2009;104:3069-79.

9. Tong JL, Ran ZH, Shen J, Xiao SD. Sequential therapy vs. standard triple therapies for Helicobacter pylori infection: a meta-analysis. J Clin Pharm Ther. 2009;34:41-53.

10. Kim JS, Kim BW, Ham JH, Park HW, Kim YK, Lee MY, Ji JS, Lee BI, Choi H. Sequential Therapy for Helicobacter pylori Infection in Korea: Systematic Review and Meta-Analysis. Gut Liver. 2013;7:546-51.

11. Zullo A, Rinaldi V, Winn S, Meddi P, Lionetti R, Hassan C, Ripani C, Tomaselli G, Attili AF. A new highly effective short-term therapy schedule for Helicobacter pylori eradication. Aliment Pharmacol Tther. 2000;14:715-8.

12. Yoon H, Lee DH, Kim N, Park YS, Shin CM, Kang KK, Oh DH, Jang DK, Chung JW. Meta-analysis: Is sequential therapy superior to standard triple therapy for Helicobacter pylori infection in Asian adults? J Gastroenterol Hepatol. 2013;28:1801-9.

13. Chung JW, Jung YK, Kim YJ, Kwon KA, Kim JH, Lee JJ, Lee SM, Hahm KB, Lee SM, Jeong JY, et al. Ten-day sequential versus triple therapy for Helicobacter pylori eradication: a prospective, open-label, randomized trial. J Gastroenterol Hepatol. 2012;27:1675-80.

14. Lee JY, Kim N. Future trends of Helicobacter pylori eradication therapy in Korea. Korean J Gastroenterol. 2014;63:158-70.

15. Kim SG, Jung HK, Lee HL, Jang JY, Lee H, Kim CG, Shin WG, Shin ES, Lee YC. Guidelines for the diagnosis and treatment of Helicobacter pylori infection in Korea, 2013 revised edition. J Gastroenterol Hepatol. 2014;29:1371-86.

16. Marin AC, MCNicholl AG, Gisbert JP. A review of rescue regimens after clarithromycin-containing triple therapy failure (for Helicobacter pylori eradication). Exp opin Pharmacotherapy. 2013:14:843-61.

17. Venerito $M$, Krieger $T$, Ecker $T$, Leandro G, Malfertheiner P. Meta-analysis of bismuth quadruple therapy versus clarithromycin triple therapy for empiric primary treatment of Helicobacter pylori infection. Digestion. 2013;88:33-45.

18. Lee BH, Kim N, Hwang TJ, Lee SH, Park YS, Hwang JH, Kim JW, Jeong SH, Lee $\mathrm{DH}$, Jung $\mathrm{HC}$, et al. Bismuth-containing quadruple therapy as secondline treatment for Helicobacter pylori infection: effect of treatment duration and antibiotic resistance on the eradication rate in Korea. Helicobacter. 2010;15:38-45

19. Lee JW, Kim N, Kim JM, Nam RH, Chang H, Kim JY, Shin CM, Park YS, Lee $\mathrm{DH}$, Jung HC. Prevalence of primary and secondary antimicrobial resistance of Helicobacter pylori in Korea from 2003 through 2012. Helicobacter. 2013;18:206-14.

20. Liang X, Xu X, Zheng Q, Zhang W, Sun Q, Liu W, Xiao S, Lu H. Efficacy of bismuth-containing quadruple therapies for clarithromycin-, metronidazole-, and fluoroquinolone-resistant Helicobacter pylori infections in a prospective study. Clin Gastroenterol Hepatol. 2013;11:802-807.e801.

21. Kadayifci A, Uygun A, Polat Z, Kantarcioglu M, Kilciler G, Baser O, Ozcan A, Emer O. Comparison of bismuth-containing quadruple and concomitant therapies as a first-line treatment option for Helicobacter pylori. Turkish J Gastroenterol. 2012;23:8-13.

22. Cetinkaya ZA, Sezikli M, Guzelbulut F, Cosgun S, Duzgun S, Kurdas OO. Comparison of the efficacy of the two tetracycline-containing sequential therapy regimens for the eradication of Helicobacter pylori: 5 days versus 14 days amoxicillin. Helicobacter. 2010;15:143-7.

23. Chi CH, Lin CY, Sheu BS, Yang HB, Huang AH, Wu JJ. Quadruple therapy containing amoxicillin and tetracycline is an effective regimen to rescue failed triple therapy by overcoming the antimicrobial resistance of Helicobacter pylori. Aliment Pharmacol Ther. 2003;18:347-53.

24. Kim SE, Park YS, Kim N, Kim MS, Jo HJ, Shin CM, Lee SH, Hwang JH, Kim JW, Jeong SH, et al. Effect of Helicobacter pylori Eradication on Functional Dyspepsia. J Neurogastroenterol Motility. 2013;19:233-43.
25. Megraud F, Coenen S, Versporten A, Kist M, Lopez-Brea M, Hirschl AM, Andersen LP, Goossens H, Glupczynski Y. Helicobacter pylori resistance to antibiotics in Europe and its relationship to antibiotic consumption. Gut. 2013:62:34-42.

26. Boyanova L, Mitov I. Geographic map and evolution of primary Helicobacter pylori resistance to antibacterial agents. Exp Rev Anti-infect Ther. 2010;8:59-70.

27. Vakil N, Vaira D. Treatment for H. pylori infection: new challenges with antimicrobial resistance. J Clin Gastroenterol. 2013;47:383-8.

28. Gomollon F, Sicilia B, Ducons JA, Sierra E, Revillo MJ, Ferrero M. Third line treatment for Helicobacter pylori: a prospective, culture-guided study in peptic ulcer patients. Aliment Pharmacol Ther. 2000;14:1335-8.

29. Perri F, Festa V, Merla A, Quitadamo M, Clemente R, Andriulli A. Amoxicillin/ tetracycline combinations are inadequate as alternative therapies for Helicobacter pylori infection. Helicobacter. 2002;7:99-104.

30. Lv ZF, Wang FC, Zheng HL, Wang B, Xie Y, Zhou XJ, Lv NH. Meta-analysis: is combination of tetracycline and amoxicillin suitable for Helicobacter pylori infection? World J Gastroenterol. 2015;21:2522-33.

31. Uygun A, Ozel AM, Yildiz O, Aslan M, Yesilova Z, Erdil A, Bagci S, Gunhan O. Comparison of three different second-line quadruple therapies including bismuth subcitrate in Turkish patients with non-ulcer dyspepsia who failed to eradicate Helicobacter pylori with a 14-day standard first-line therapy. J Gastroenterol Hepatol. 2008:23:42-5.

32. Malfertheiner P, Bazzoli F, Delchier JC, Celinski K, Giguere M, Riviere M, Megraud F. Helicobacter pylori eradication with a capsule containing bismuth subcitrate potassium, metronidazole, and tetracycline given with omeprazole versus clarithromycin-based triple therapy: a randomised, openlabel, non-inferiority, phase 3 trial. Lancet. 2011;377(9769):905-13.

33. Levison ME. Pharmacodynamics of antimicrobial agents. Bactericidal and postantibiotic effects. Infect Dis Clin North Am. 1995;9:483-95.

34. Adir J, Barr WH. Dose-dependent bioavailability of tetracycline in man. J Pharmacokinet Biopharm. 1978;6:99-110.

35. Liu P, Muller M, Derendorf H. Rational dosing of antibiotics: the use of plasma concentrations versus tissue concentrations. Intern J Antimicrob Agents. 2002;19:285-90.

\section{Submit your next manuscript to BioMed Central and we will help you at every step:}

- We accept pre-submission inquiries

- Our selector tool helps you to find the most relevant journal

- We provide round the clock customer support

- Convenient online submission

- Thorough peer review

- Inclusion in PubMed and all major indexing services

- Maximum visibility for your research

Submit your manuscript at www.biomedcentral.com/submit
) Biomed Central 